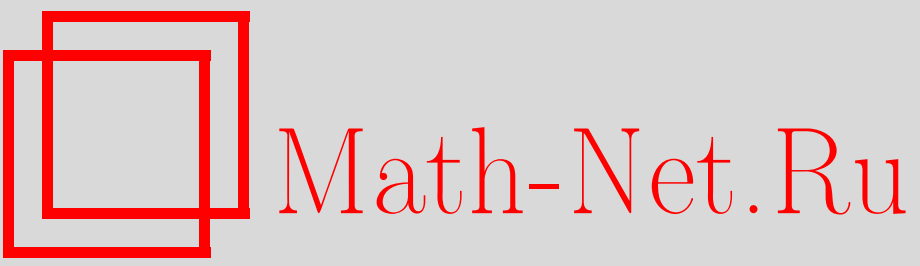

В. А. Копытцев, Многомерная теорема Пуассона для чисел решений случайных включений, близких к заданным векторам, Матем. вопр. криптогр., 2016 , том 7, выпуск 4, 67-80

DOI: https://doi.org/10.4213/mvk204

Использование Общероссийского математического портала Math-Net.Ru подразумевает, что вы прочитали и согласны с пользовательским соглашением

http: //www . mathnet.ru/rus/agreement

Параметры загрузки :

IP : 54.197 .217 .227

26 апреля 2023 г., 12:29:06 
МАТЕМАТИЧЕСКИЕ ВОПРОСЫ КРИПТОГРАФИИ

2016 T. 7 № 4 C. $67-80$

УДК 519.212.2

\title{
Многомерная теорема Пуассона для чисел решений случайных включений, близких к заданным векторам
}

\author{
В. А. Копытцев
}

\author{
Академия криптографии Российской Федерации, Москва
}

Получено 01.ІХ.2016

\begin{abstract}
Аннотация. Рассматривается число решений случайного включения над конечным полем, отличающихся от некоторого опорного вектора не более чем в заданном числе координат. Приводятся условия, при которых числа решений, связанные с несколькими опорными векторами, асимптотически независимы, а их распределения сходятся к пуассоновским распределениям, когда размерность векторов стремится к бесконечности.
\end{abstract}

Ключевые слова: случайные включения над конечным полем, число решений, пуассоновские аппроксимации для чисел решений

\section{A multivariate Poisson theorem for the number of solutions of random inclusions close to given vectors}

\section{V.A. Kopytcev \\ Academy of Cryptography of the Russian Federation, Moscow}

\begin{abstract}
We consider the number of solutions of random inclusion over a finite field that differ from a reference vector by no more than a specified number of coordinates. We find conditions on the growth of vector dimensions under which the number of solutions close to some reference vectors are asymptotically independent and their distributions converge to the Poisson distributions.
\end{abstract}

Keywords: random inclusions over the finite field, number of solutions, Poisson approcsimation for the number of solutions 


\section{1. Введение}

Рассмотрим над конечным полем $K=G F(q)$ систему включений

$$
x \in S_{r}(z), \quad A x+G(x) \in B(x),
$$

где $S_{r}(z)=\left\{x \in K^{n}: 1 \leqslant\|x-z\| \leqslant r\right\}$ - множество векторов пространства $K^{n}$, отличающихся от вектора $z \in K^{n}$ не более чем в заданном числе $r$ координат; $A$ - случайная матрица размера $T \times n ; G-$ случайное отображение $K^{n}$ в $K^{T}$, не зависящее от матрицы $A$. Множество $B(x)$ векторов размерности $T$ определяется значением вектора $x \in K^{n}$.

Вместе с системой (1) рассмотрим систему включений

$$
x \in X_{r}(z), \quad A x+G(x) \in B(x),
$$

где $X_{r}(z)=\left\{x \in K^{n}:\|x-z\|=r\right\}$.

Далее предполагается, что элементы матрицы $A=\left\|a_{i, j}\right\|_{T \times n}$ независимы в совокупности и имеют распределение

$$
\mathbf{P}\left\{a_{i, j}=k\right\}=\frac{1+\Delta_{i, j}(k)}{q}, \quad k \in K,
$$

где $q=|K|$ и $\sum_{k \in K} \Delta_{i, j}(k)=0, i=1, \ldots, T, j=1, \ldots, n$. Пусть

$$
\Delta=\max _{i, j, k}\left|\Delta_{i, j}(k)\right|<1 .
$$

Обозначим $\xi\left(S_{r}(z), A+G, \bar{B}\right)$ и $\xi\left(X_{r}(z), A+G, \bar{B}\right)$ число решений системы включений (1) и системы включений (2) соответственно (под $\bar{B}$ понимается набор множеств $\left.B(x), x \in K^{n}\right)$. Распределения этих величин исследовались в работах $[1,2]$, где приведены достаточные условия для их сходимости к распределению Пуассона.

В данной работе исследуются совместное распределение величин $\xi\left(S_{r}\left(z^{s}\right), A+G, \bar{B}\right), \quad s=1, \ldots, h$, и совместное распределение величин $\xi\left(X_{r}\left(z^{s}\right), A+G, \bar{B}\right), \quad s=1, \ldots, h$, связанных с векторами $z^{1}, \ldots, z^{h}$ пространства $K^{n}$. Частный случай, когда $G(x) \equiv 0^{T}$ и $B(x)=b \in K^{T}$ для всех $x \in K^{n}$, рассмотрен в [3].

Положим

$$
J(s)=\bigcup_{x \in X_{r}\left(z^{s}\right)}\{(x, b): b \in B(x)\}, \quad s=1, \ldots, h .
$$


Для обозначения предельных соотношений вида

$$
\mathbf{P}\left\{\xi_{1}=k_{1}, \ldots, \xi_{h}=k_{h}\right\} \rightarrow \frac{\lambda_{1}^{k_{1}} e^{-\lambda_{1}}}{k_{1} !} \cdots \frac{\lambda_{h}^{k_{h}} e^{-\lambda_{h}}}{k_{h} !}, \quad k_{1}, \ldots, k_{h}=0,1, \ldots
$$

будем использовать запись $\left(\xi_{s}, s=1, \ldots, h\right) \rightarrow \Pi\left(\lambda_{1}\right) \cdots \Pi\left(\lambda_{h}\right)$.

Теорема 1. Пусть $K=G F(q), \operatorname{rank}\left(z^{1}, \ldots, z^{h}\right)=h$, распределение элементов матрицы А удовлетворяет условиям (3), (4), $0^{T} \notin B(x)$ при всех $x \in S_{r}\left(z^{s}\right), s=1, \ldots, h$, и при $n, T \rightarrow \infty$ выполнены соотношения $r=o(n)$, $T \Delta=o(1)$,

$$
\begin{gathered}
\max _{x \in S_{r}\left(z^{s}\right)}|B(x)| / \min _{x \in S_{r}\left(z^{s}\right)}|B(x)|=O(1), \quad s=1, \ldots, h, \\
\frac{\mid J(s)) \mid}{q^{T}} \rightarrow \lambda_{s}, \quad 0<\lambda_{s}<\infty, \quad s=1, \ldots, h .
\end{gathered}
$$

Тогда

$$
\begin{gathered}
\left(\xi\left(S_{r}\left(z^{s}\right), A+G, \bar{B}\right), s=1, \ldots, h\right) \rightarrow \Pi\left(\lambda_{1}\right) \cdots \Pi\left(\lambda_{h}\right), \\
\left(\xi\left(X_{r}\left(z^{s}\right), A+G, \bar{B}\right), s=1, \ldots, h\right) \rightarrow \Pi\left(\lambda_{1}\right) \cdots \Pi\left(\lambda_{h}\right), \\
\mathbf{P}\left\{\xi\left(S_{r}\left(z^{s}\right), A+G, \bar{B}\right)=\xi\left(X_{r}\left(z^{s}\right), A+G, \bar{B}\right), s=1, \ldots, h\right\} \rightarrow 1 .
\end{gathered}
$$

Обозначим $\xi\left(S_{r}\left(z^{1}, \ldots, z^{h}\right), A+G, \bar{B}\right)$ число решений системы включений

$$
x \in S_{r}\left(z^{1}, \ldots, z^{h}\right), \quad A x+G(x) \in B(x),
$$

где

$$
S_{r}\left(z^{1}, \ldots, z^{h}\right)=\bigcup_{s=1}^{h} S_{r}\left(z^{s}\right) .
$$

Аналогично определим величину $\xi\left(X_{r}\left(z^{1}, \ldots, z^{h}\right), A+G, \bar{B}\right)$, где

$$
X_{r}\left(z^{1}, \ldots, z^{h}\right)=\bigcup_{s=1}^{h} X_{r}\left(z^{s}\right)
$$

Теорема 2. Пусть выполнены условия теоремы 1. Тогда

$$
\mathbf{P}\left\{\xi\left(S_{r}\left(z^{1}, \ldots, z^{h}\right), A+G, \bar{B}\right)=\sum_{i=1}^{h} \xi\left(S_{r}\left(z^{i}\right), A+G, \bar{B}\right)\right\} \rightarrow 1 .
$$


Следствие 1. Пусть выполнены условия теоремы 1. Тогда

$$
\begin{gathered}
\xi\left(S_{r}\left(z^{1}, \ldots, z^{h}\right), A+G, \bar{B}\right) \rightarrow \Pi\left(\lambda_{1}+\cdots+\lambda_{h}\right), \\
\xi\left(X_{r}\left(z^{1}, \ldots, z^{h}\right), A+G, \bar{B}\right) \rightarrow \Pi\left(\lambda_{1}+\cdots+\lambda_{h}\right) .
\end{gathered}
$$

В разделе 2 приведены леммы из [1,3], которые в разделе 3 используются в доказательстве теоремы 1. В разделе 4 приведено доказательство теоремы 2.

\section{2. Вспомогательные утверждения}

Пусть $F(x)=A x+G(x)$, где $(T \times n)$-матрица $A$ не зависит от отображения $G: K^{n} \rightarrow K^{T}$.

Лемма 1. Пусть $\left(x^{1}, \ldots, x^{k}\right) \in K^{n}, b^{1}, \ldots, b^{k} \in K^{T}$ и выполнены условия (3), (4). Тогда при всех $k=1,2, \ldots$

$$
\mathbf{P}\left\{F\left(x^{1}\right)=b^{1}, \ldots, F\left(x^{k}\right)=b^{k}\right\} \leqslant\left(\frac{1+\Delta}{q}\right)^{j T},
$$

если $1 \leqslant \operatorname{rank}\left(x^{1}, \ldots, x^{k}\right)=j \leqslant k-1$, а если $\operatorname{rank}\left(x^{1}, \ldots, x^{k}\right)=k$, то

$$
\left(\frac{1-\Delta}{q}\right)^{k T} \leqslant \mathbf{P}\left\{F\left(x^{1}\right)=b^{1}, \ldots, F\left(x^{k}\right)=b^{k}\right\} \leqslant\left(\frac{1+\Delta}{q}\right)^{k T} .
$$

Лемма 1 является следствием леммы 1 [1] и условия о независимости матрицы $A$ и отображения $G$ (отметим, что лемма 1 [1] есть частный вариант леммы 1 , соответствующий условию $G \equiv 0^{T}$ ).

Пусть заданы $h$ комплектов векторов

$$
y^{1, s}, \ldots, y^{k(s), s} \in Y_{r}, \quad s=1, \ldots, h,
$$

где $Y_{r}=\left\{y \in K^{n}:\|y\|=r\right\}, r \geqslant 1$. Пусть

$$
\sum_{s=1}^{h} k(s) \geqslant 2, \quad k(s) \geqslant 0, \quad s=1, \ldots, h .
$$

При $k(s)=0$ полагаем, что комплект с номером $s$ пустой. В каждом непустом комплекте все векторы различны.

Комплектам из набора (19) сопоставим комплекты векторов

$$
x^{1, s}, \ldots, x^{k(s), s} \in K^{n}, \quad s=1, \ldots, h,
$$

где $x^{i, s}=z^{s}+y^{i, s}, \quad i=1, \ldots, k(s)$. 
Определим некоторую сквозную нумерацию векторов в комплектах (20). Пусть

$$
\left\{y^{1, s}, \ldots, y^{k(s), s}, s=1, \ldots, h\right\}=\left\{y^{1}, \ldots, y^{k}\right\},
$$

где $k=k(1)+\cdots+k(h)$. Используя эту нумерацию, занумеруем все векторы в комплектах (20):

$$
\left\{x^{1, s}, \ldots, x^{k(s), s}, s=1, \ldots, h\right\}=\left\{x^{1}, \ldots, x^{k}\right\} .
$$

Для векторов $y^{1}, \ldots, y^{k} \in Y_{r}$ положим

$$
\begin{gathered}
M\left(y^{1}, \ldots, y^{k}\right)=\bigcup_{j=1}^{k}\left\{i \in\{1, \ldots, n\}: y_{i}^{j} \neq 0\right\}, \\
\mu\left(y^{1}, \ldots, y^{k}\right)=\left|M\left(y^{1}, \ldots, y^{k}\right)\right| .
\end{gathered}
$$

Пусть $\bar{z}^{s}=\left(\bar{z}_{1}^{s}, \ldots, \bar{z}_{n}^{s}\right), \quad s=1, \ldots, h$, где

$$
\bar{z}_{i}^{s}= \begin{cases}z_{i}^{s}, & \text { если } i \notin M\left(y^{1}, \ldots, y^{k}\right), \\ \bar{z}_{i}^{s}=0 & \text { в противном случае. }\end{cases}
$$

Лемма 2. Пусть $k \geqslant 2, \operatorname{rank}\left(\bar{z}^{1}, \ldots, \bar{z}^{h}\right)=h$, при целом $2 \leqslant j \leqslant k$ выполнено неравенство

$$
\mu\left(y^{1}, \ldots, y^{k}\right)>r(j-1)-r / 2 \text {. }
$$

Тогда

$$
\operatorname{rank}\left(x^{1}, \ldots, x^{k}\right) \geqslant j .
$$

Эта лемма следует из леммы 8 [3] (при $m(0)=0$ в обозначениях [3]).

Положим

$$
R_{k}^{(j)}=\frac{r}{q^{j T}} C_{n}^{r j-\omega(r)}\left(C_{r j-\omega(r)}^{r}\right)^{k}(q-1)^{r k} h^{k},
$$

где $\omega(1)=1$ и $\omega(r)=[r / 2]$ при $r \geqslant 2,[a]-$ целая часть числа $a$.

Лемма 3. Существует такая константа $C<\infty$, что при всех $n, T, r, h \geqslant$ $1, k \geqslant 2, r k \leqslant n / 2,2 \leqslant j \leqslant k$ выполнено неравенство

$$
R_{k}^{(j)} \leqslant \Lambda^{j} \exp \{-\omega(r) \ln (n / r)+r k(\ln k+C)\},
$$

где $\Lambda=C_{n}^{r}(q-1)^{r} q^{-T}$.

Эта лемма повторяет лемму 10 [3]. 


\section{3. Доказательство теоремы 1}

Используя выражение (5) для $J(s)$, получаем:

$$
\min _{\left.x \in S_{r}\left(z^{s}\right)\right)}|B(x)| \leqslant \frac{|J(s)|}{C_{n}^{r}(q-1)^{r}} \leqslant \max _{x \in S_{r}\left(z^{s}\right)}|B(x)| .
$$

Поэтому из условий (6), (7) следует, что при любом значении $s=1, \ldots, h$ справедлива оценка

$$
\frac{1}{q^{T}} C_{n}^{r}(q-1)^{r} \max _{x \in S_{r}\left(z^{s}\right)}|B(x)|=O(1) .
$$

С учетом (27) получаем:

$$
\begin{gathered}
\mathbf{E}\left(\xi\left(S_{r}\left(z^{s}\right), A+G, \bar{B}\right)-\xi\left(X_{r}\left(z^{s}\right), A+G, \bar{B}\right)\right)=\mathbf{E}\left(\xi\left(S_{r-1}\left(z^{s}\right), A+G, \bar{B}\right) \leqslant\right. \\
\leqslant\left(\frac{1+\Delta}{q}\right)^{T} \max _{x \in S_{r}\left(z^{s}\right)}|B(x)| \sum_{i=0}^{r-1} C_{n}^{i}(q-1)^{i}= \\
=o\left(q^{-T} C_{n}^{r}(q-1)^{r} \max _{x \in S_{r}\left(z^{s}\right)}|B(x)|\right)=o(1), \quad s=1, \ldots, h .
\end{gathered}
$$

Значит, верно соотношение (10), и достаточно показать, что выполнено (9).

Пусть $A\left(z^{1}, \ldots, z^{h}\right)$ - матрица размера $n$ на $h$, составленная из векторовстолбцов $z^{1}, \ldots, z^{h}$. Согласно условиям теоремы можно полагать, что $n>h$. Выберем в матрице $A\left(z^{1}, \ldots, z^{h}\right)$ произвольные $h$ линейно независимых строк (такие строки найдутся, так как по условию $\operatorname{rank}\left(z^{1}, \ldots, z^{h}\right)=h$ ). Пусть $i_{1}, \ldots, i_{h}-$ номера выбранных строк. При $r \geqslant 1$ положим

$$
\begin{gathered}
Y_{r, h}^{\prime}=\left\{y \in Y_{r}: i_{1}, \ldots, i_{h} \notin M(y)\right\}, \\
Y_{r, h}^{\prime \prime}=Y_{r} \backslash Y_{r, h}^{\prime},
\end{gathered}
$$

где $Y_{r}=\left\{y \in K^{n}:\|y\|=r\right\}, M(y)=\left\{i \in\{1, \ldots, n\}: y_{i} \neq 0\right\}$.

Используя множества (29), (30), получаем

$$
\xi\left(X_{r}\left(z^{s}\right), A+G, \bar{B}\right)=\xi^{\prime}(s)+\xi^{\prime \prime}(s), \quad s=1, \ldots, h,
$$

где

$$
\xi^{\prime}(s)=\sum_{(x, b) \in J(s), x-z^{s} \in Y_{r, h}^{\prime}} I\{A x+G(x)=b\}
$$




$$
\xi^{\prime \prime}(s)=\sum_{(x, b) \in J(s), x-z^{s} \in Y_{r, h}^{\prime \prime}} I\{A x+G(x)=b\},
$$

$I\{C\}$ - индикатор события $C$. Покажем, что

$$
\left(\xi^{\prime}(s), s=1, \ldots, h\right) \rightarrow \Pi\left(\lambda_{1}\right) \cdots \Pi\left(\lambda_{h}\right),
$$

(величины $\lambda_{1}, \ldots, \lambda_{h}$ в (34) определяются формулами (7)) и

$$
\mathbf{P}\left\{\xi^{\prime \prime}(s)=0, s=1, \ldots, h\right\} \rightarrow 1 .
$$

Из соотношений (34) - (35) будет следовать (9).

Сначала докажем (34). Положим

$$
\begin{gathered}
J^{\prime}=\bigcup_{s \in\{1, \ldots, h\}}\left\{(x, b) \in J(s): x-z(s) \in Y_{r, h}^{\prime}\right\}, \\
J_{k}^{\prime}=\left\{\left(\left(x^{1}, b^{1}\right), \ldots,\left(x^{k}, b^{k}\right)\right) \in\left(J^{\prime}\right)^{k}:\left(x^{\alpha}, b^{\alpha}\right) \neq\left(x^{\beta}, b^{\beta}\right)(\alpha \neq \beta)\right\} .
\end{gathered}
$$

Согласно теореме 1 [4] (теореме 3 [5]) для доказательства соотношения (34) достаточно показать, что (используем обозначение $F(x)=A x+G(x)$ )

$$
\begin{gathered}
\sum_{(x, b) \in J(s), x-z^{s} \in Y_{r, h}^{\prime}} \mathbf{P}\{F(x)=b\} \rightarrow \lambda(s), s=1, \ldots, h, \\
\max _{(x, b) \in J^{\prime}} \mathbf{P}\{F(x)=b\} \rightarrow 0
\end{gathered}
$$

и при всех $k=2,3, \ldots$ (используем обозначение $v^{i}=\left(x^{i}, b^{i}\right)$ )

$$
\begin{aligned}
& \sum_{\left(v^{1}, \ldots, v^{k}\right) \in J_{k}^{\prime}} \mid \mathbf{P}\left\{F\left(x^{1}\right)=b^{1}, \ldots,\right.\left.F\left(x^{k}\right)=b^{k}\right\}- \\
& \quad-\mathbf{P}\left\{F\left(x^{1}\right)=b^{1}\right\} \cdots \mathbf{P}\left\{F\left(x^{k}\right)=b^{k}\right\} \mid \rightarrow 0 .
\end{aligned}
$$

Проверим условия (37) - (39). Сначала докажем соотношения (37).

Используя при $k=1$ неравенства (18), получаем

$$
\left(\frac{1-\Delta}{q}\right)^{T} R(s) \leqslant \sum_{(x, b) \in J(s), x-z^{s} \in Y_{r, h}^{\prime}} \mathbf{P}\{F(x)=b\} \leqslant\left(\frac{1+\Delta}{q}\right)^{T} R(s),
$$


где $s=1, \ldots, h$ и $R(s)=|J(s)|-\bar{R}(s)$,

$$
\begin{gathered}
\bar{R}(s)=\left|\left\{(x, b) \in J(s), x-z^{s} \notin Y_{r, h}^{\prime}\right\}\right| \leqslant \\
\leqslant\left|\left\{x \in X_{r}\left(z^{s}\right), x-z^{s} \notin Y_{r, h}^{\prime}\right\}\right| \max _{x \in S_{r}\left(z^{s}\right)}|B(x)| .
\end{gathered}
$$

Из условия $T \Delta \rightarrow 0$ следует, что

$$
(1 \pm \Delta)^{T} \rightarrow 1
$$

Вместе с этим

$$
\frac{\left|\left\{x \in X_{r}\left(z^{s}\right), x-z^{s} \notin Y_{r, h}^{\prime}\right\}\right|}{\left|X_{r}\left(z^{s}\right)\right|}=\frac{\sum_{j=1}^{h} C_{h}^{j} C_{n-h}^{r-j}(q-1)^{r}}{C_{n}^{r}(q-1)^{r}} \rightarrow 0
$$

и согласно (6), (7)

$$
\frac{\left|X_{r}\left(z^{s}\right)\right|}{q^{T}} \max _{x \in S_{r}\left(z^{s}\right)}|B(x)| \leqslant \frac{|J(s)|}{q^{T}} \frac{\max _{x \in S_{r}\left(z^{s}\right)}|B(x)|}{\min _{x \in S_{r}\left(z^{s}\right)}|B(x)|}=O(1) .
$$

Из (40) - (43) и соотношения (7) получаем (37).

Проверим (38): в силу (18) (где полагаем $k=1$ ) и (41) получаем

$$
\max _{(x, b) \in J^{\prime}} \mathbf{P}\{F(x)=b\} \leqslant\left(\frac{1+\Delta}{q}\right)^{T} \rightarrow 0 .
$$

Осталось проверить (39). Для этого воспользуемся леммой 2 (см. соотношения (21), (22) и следующие). Условие $\operatorname{rank}\left(\bar{z}^{1}, \ldots, \bar{z}^{h}\right)=h$ этой леммы выполнено, так как матрица $A\left(\bar{z}^{1}, \ldots, \bar{z}^{h}\right)$, составленная из векторов-столбцов $\bar{z}^{1}, \ldots, \bar{z}^{h}$, согласно определению (29) множества $Y_{r, h}^{\prime}$ содержит линейно независимые строки с номерами $i_{1}, \ldots, i_{h}$.

Разобьем сумму из условия (39) на две части, полагая (в сокращенной записи)

$$
\sum_{\left(v^{1}, \ldots, v^{k}\right) \in J_{k}^{\prime}}=\sum_{\substack{\left(v^{1}, \ldots, v^{k}\right) \in J_{k}^{\prime} \\
\mu\left(y^{1}, \ldots, y^{k}\right) \leqslant r k-r / 2}}+\sum_{\begin{array}{c}
\left(v^{1}, \ldots, v^{k}\right) \in J_{k}^{\prime} \\
\mu\left(y^{1}, \ldots, y^{k}\right)>r k-r / 2
\end{array}}
$$

Сначала оценим вторую сумму в правой части (44). В случае

$$
\mu\left(y^{1}, \ldots, y^{k}\right)>r k-\frac{r}{2}
$$


согласно лемме 2 (см. (23), (24)) $\operatorname{rank}\left(x^{1}, \ldots, x^{k}\right)=k$. Значит, справедливы неравенства (18) и

$$
\begin{aligned}
\mid \mathbf{P}\left\{F\left(x^{1}\right)=b^{1}, \ldots, F\left(x^{k}\right)=\right. & \left.b^{k}\right\}-\mathbf{P}\left\{F\left(x^{1}\right)=b^{1}\right\} \cdots \mathbf{P}\left\{F\left(x^{k}\right)=b^{k}\right\} \mid= \\
& =q^{-k T} O(T \Delta),
\end{aligned}
$$

где по условию $T \Delta \rightarrow 0$. Поэтому

$$
\sum_{\substack{\left(v^{1}, \ldots, v^{k}\right) \in J_{k}^{\prime} \\ \mu\left(y^{1}, \ldots, y^{k}\right)>r k-r / 2}}=o\left(\left(\lambda_{1}+\cdots+\lambda_{h}\right)^{k}\right) \rightarrow 0 .
$$

Для первой суммы в правой части (44) имеем

$$
\sum_{\substack{\left(v^{1}, \ldots, v^{k}\right) \in J_{k}^{\prime} \\ \mu\left(y^{1}, \ldots, y^{k}\right) \leqslant r k-r / 2}} \leqslant \Sigma_{1}+\Sigma_{2}
$$

где

$$
\begin{aligned}
\Sigma_{1}= & \sum_{\substack{\left(v^{1}, \ldots, v^{k}\right) \in J_{k}^{\prime} \\
\mu\left(y^{1}, \ldots, y^{k}\right) \leqslant r k-r / 2}} \mathbf{P}\left\{F\left(x^{1}\right)=b^{1}, \ldots, F\left(x^{k}\right)=b^{k}\right\}, \\
\Sigma_{2}= & \sum_{\substack{\left(v^{1}, \ldots, v^{k}\right) \in J_{k}^{\prime} \\
\mu\left(y^{1}, \ldots, y^{k}\right) \leqslant r k-r / 2}} \mathbf{P}\left\{F\left(x^{1}\right)=b^{1}\right\} \cdots \mathbf{P}\left\{F\left(x^{k}\right)=b^{k}\right\} .
\end{aligned}
$$

Оценим суммы (47), (48). Сопоставим набору векторов $x^{1}, \ldots, x^{k}$ систему $L_{x}\left(x^{1}, \ldots, x^{k}\right)$ однородных линейных уравнений вида

$$
\alpha_{1} x^{1}+\cdots+\alpha_{k} x^{k}=0^{n}
$$

(возможно, пустую), составленную из всех линейных соотношений, которым удовлетворяет набор $x^{1}, \ldots, x^{k}$. Системе $L_{x}\left(x^{1}, \ldots, x^{k}\right)$ сопоставим идентичную по составу систему $L_{b}\left(x^{1}, \ldots, x^{k}\right)$ из линейных уравнений относительно $b^{1}, \ldots, b^{k} \in K^{T}$. 
Пусть $g-$ некоторая реализация отображения $G$. Обозначим через $B_{g}\left(x^{1}, \ldots, x^{k}\right)$ множество решений $\left(b^{1}, \ldots, b^{k}\right)$ системы $L_{b}\left(x^{1}, \ldots, x^{k}\right)$, удовлетворяющих условию

$$
b^{1} \in B\left(x^{1}\right)-g\left(x^{1}\right), \ldots, b^{k} \in B\left(x^{k}\right)-g\left(x^{k}\right),
$$

где

$$
B(x)-g(x)=\left\{b-g(x) \in K^{T}: b \in B(x)\right\} .
$$

Если

$$
\operatorname{rank}\left(x^{1}, \ldots, x^{k}\right)=k
$$

(т. е. система $L_{b}\left(x^{1}, \ldots, x^{k}\right)$ пустая), то $B_{g}\left(x^{1}, \ldots, x^{k}\right)$ есть множество векторов, удовлетворяющих условию (49).

При $k \geqslant 2$ положим

$$
D_{k}^{\prime}=\left\{\left(x^{1}, \ldots, x^{k}\right) \in\left(D^{\prime}\right)^{k}: x^{\alpha} \neq x^{\beta}(\alpha \neq \beta)\right\}
$$

где

$$
D^{\prime}=\bigcup_{s=1}^{h}\left\{x \in K^{n}: x-z^{s} \in Y_{r, h}^{\prime}\right\} .
$$

Используя определения (36), (50), из (47) получаем

$$
\begin{aligned}
& \Sigma_{1} \leqslant \sum_{g} \mathbf{P}\{G=g\} \times \\
& \times \sum_{j=2}^{k} \sum_{\substack{\left(x^{1}, \ldots, x^{k}\right) \in D_{k}^{\prime} \\
r(j-1)-r / 2<\mu\left(y^{1}, \ldots, y^{k}\right) \leqslant r j-r / 2}} \sum_{\left(b^{1}, \ldots, b^{k}\right) \in B_{g}\left(x^{1}, \ldots, x^{k}\right)} \mathbf{P}\left\{A x^{1}=b^{1}, \ldots, A x^{k}=b^{k}\right\},
\end{aligned}
$$

где сумма $\sum_{g}$ проводится по всем реализациям отображения $G$. Положим

$$
m(B)=\max _{x \in \bigcup_{s=1}^{h} S\left(z^{s}\right)}|B(x)| .
$$

Лемма 4. Пусть $2 \leqslant j \leqslant k,\left(x^{1}, \ldots, x^{k}\right) \in D_{k}^{\prime}, \mu\left(y^{1}, \ldots, y^{k}\right)>r(j-1)-r / 2$ $u$

$$
q \cdot m(B)\left(\frac{1+\Delta}{q}\right)^{T} \leqslant 1
$$


Тогда при любой реализаиии $g$ отображения $G$

$$
\sum_{\left(b_{1}, \ldots, b_{k}\right) \in B_{g}\left(x_{1}, \ldots, x_{k}\right)} \mathbf{P}\left\{A x^{1}=b^{1}, \ldots, A x^{k}=b^{k}\right\} \leqslant\left(\frac{m(B)}{q^{T-1}}\right)^{j}(1+\Delta)^{j T} .
$$

Доказательство. Пусть

$$
2 \leqslant \operatorname{rank}\left(x^{1}, \ldots, x^{k}\right)=i \leqslant k .
$$

Не ограничивая общности, положим $\operatorname{rank}\left(x^{1}, \ldots, x^{i}\right)=i$. Тогда в каждом наборе $\left(b^{1}, \ldots, b^{k}\right) \in B_{g}\left(x^{1}, \ldots, x^{k}\right)$ любой вектор линейно выражается через векторы $b^{1}, \ldots, b^{i}$. Поэтому

$$
\left|B_{g}\left(x^{1}, \ldots, x^{k}\right)\right| \leqslant(q \cdot m(B))^{i} .
$$

Вместе с этим, согласно лемме 1 (см. (17), (18))

$$
\mathbf{P}\left\{A x^{1}=b^{1}, \ldots, A x^{k}=b^{k}\right\} \leqslant\left(\frac{1+\Delta}{q}\right)^{i T}
$$

и согласно лемме 2 (см. (23), (24)) из условия

$$
r(j-1)-\frac{r}{2}<\mu\left(y^{1}, \ldots, y^{k}\right)
$$

следует, что

$$
\operatorname{rank}\left(x^{1}, \ldots, x^{k}\right)=i \geqslant j .
$$

Теперь из (55), (56) и (53), (57) получаем (54).

Лемма 4 доказана.

В условиях теоремы неравенство (53), очевидно, выполнено. Положим

$$
W_{k}(l)=\left|\left\{\left(x^{1}, \ldots, x^{k}\right) \in D_{k}^{\prime}: \mu\left(y^{1}, \ldots, y^{k}\right)=l\right\}\right| .
$$

В (58) имеем $x^{i}=z+y^{i}$, где $z \in\left\{z^{1}, \ldots, z^{h}\right\}$. Поэтому

$$
\begin{aligned}
W_{k}(l) & \leqslant h^{k}\left|\left\{\left(y^{1}, \ldots, y^{k}\right) \in\left(Y_{r}\right)^{k}: \mu\left(y^{1}, \ldots, y^{k}\right)=l\right\}\right| \leqslant \\
& \leqslant h^{k} C_{n}^{l}\left(C_{l}^{r}(q-1)^{r}\right)^{k} .
\end{aligned}
$$


Используя (54) и (59), из (51) получаем

$$
\begin{gathered}
\Sigma_{1} \leqslant \sum_{j=2}^{k}\left(\frac{m(B)}{q^{T-1}}\right)^{j}(1+\Delta)^{j T} \sum_{r(j-1)-r / 2<l \leqslant r j-r / 2} W_{k}(l) \leqslant \\
\leqslant(1+\Delta)^{k T} \sum_{j=2}^{k}(q \cdot m(B))^{j} R_{k}^{(j)},
\end{gathered}
$$

где $R_{k}^{(j)}$ определяется по формуле (25). Из (26) вытекает оценка $R_{k}^{(j)}=o\left(\Lambda^{j}\right)$, а согласно (27) имеем $\Lambda m(B)=O(1)$. Следовательно, $\Sigma_{1} \rightarrow 0$.

Для суммы (48) выполняется оценка, аналогичная оценке (60):

$$
\begin{gathered}
\Sigma_{2} \leqslant(q \cdot m(B))^{k}\left(\frac{1+\Delta}{q}\right)^{k T} \sum_{j=2}^{k} \sum_{r(j-1)-r / 2<l \leqslant r j-r / 2} W_{k}(l) \leqslant \\
\leqslant(1+\Delta)^{k T} \sum_{j=2}^{k}(q \cdot m(B))^{j} R_{k}^{(j)} .
\end{gathered}
$$

Следовательно, $\Sigma_{2} \rightarrow 0$. Таким образом, соотношение (34) доказано.

Осталось доказать соотношение (35). Согласно (30) вектор $y=x-z^{s}$ в (33) принимает не более $\sum_{i=1}^{\min \{r, h\}} C_{h}^{i} C_{n-h}^{r-i}(q-1)^{r}$ значений. Поэтому с учетом (5), (18), (52) имеем

$$
\begin{aligned}
\mathbf{E} \xi^{\prime \prime}(s) & \leqslant \sum_{i=1}^{\min \{r, h\}} C_{h}^{i} C_{n-h}^{r-i}(q-1)^{r} m(B)\left(\frac{1+\Delta}{q}\right)^{T} \leqslant \\
& \leqslant \frac{h r}{n} C_{n}^{r}(q-1)^{r} m(B)\left(\frac{1+\Delta}{q}\right)^{T}=O\left(\frac{r}{n}\right) .
\end{aligned}
$$

Отсюда, учитывая условие $r=o(n)$, получаем, что $\mathbf{E} \xi^{\prime \prime}(s) \rightarrow 0$.

Теорема 1 доказана.

\section{4. Доказательство теоремы 2}

Отметим, что

$$
\sum_{i=1}^{h} \xi\left(S_{r}\left(z^{i}\right), A+G, \bar{B}\right) \geqslant \xi\left(S_{r}\left(z^{1}, \ldots, z^{h}\right), A+G, \bar{B}\right),
$$


при этом

$$
\begin{gathered}
\mathbf{E}\left(\sum_{i=1}^{h} \xi\left(S_{r}\left(z^{i}\right), A+G, \bar{B}\right)-\xi\left(S_{r}\left(z^{1}, \ldots, z^{h}\right), A+G, \bar{B}\right)\right) \leqslant \\
\leqslant m(B)\left(\frac{1+\Delta}{q}\right)^{T} \sum_{1 \leqslant i_{1}<i_{2} \leqslant h}\left|X_{r}\left(z^{i_{1}}\right) \cap X_{r}\left(z^{i_{2}}\right)\right|,
\end{gathered}
$$

где величина $m(B)$ определена формулой (52). Оценим правую часть неравенства (63). Для векторов $z^{i_{1}}, z^{i_{2}} \in\left\{z^{1}, \ldots, z^{h}\right\}, i_{1} \neq i_{2}$, выберем индекс

$$
j \in\{1, \ldots, n\}: z_{j}^{i_{1}} \neq z_{j}^{i_{2}} .
$$

Положим

$$
\begin{aligned}
& X_{r}^{(1, j)}\left(z^{i}\right)=\left\{x \in K^{n} \leqslant\left\|x-z^{i}\right\|=r, x_{j}=z_{j}^{i}\right\} \\
& X_{r}^{(2, j)}\left(z^{i}\right)=\left\{x \in K^{n}:\left\|x-z^{i}\right\|=r, x_{j} \neq z_{j}^{i}\right\} .
\end{aligned}
$$

Ясно, что

$$
\begin{aligned}
& X_{r}^{(1, j)}\left(z^{i}\right) \cup X_{r}^{(2, j)}\left(z^{i}\right)=X_{r}\left(z^{i}\right), \\
& X_{r}^{(1, j)}\left(z^{i}\right) \cap X_{r}^{(2, j)}\left(z^{i}\right)=\varnothing .
\end{aligned}
$$

С учетом (67), (68) получаем

$$
\left|X_{r}\left(z^{i_{1}}\right) \cap X_{r}\left(z^{i_{2}}\right)\right|=\sum_{s=1}^{2} \sum_{l=1}^{2}\left|X_{r}^{(s, j)}\left(z^{i_{1}}\right) \cap X_{r}^{(l, j)}\left(z^{i_{2}}\right)\right| .
$$

Из (64) и (65) следует равенство $\left|X_{r}^{(1, j)}\left(z^{i_{1}}\right) \cap X_{r}^{(2, j)}\left(z^{i_{2}}\right)\right|=0$. Поэтому

$$
\begin{aligned}
& \left|X_{r}\left(z^{i_{1}}\right) \cap X_{r}\left(z^{i_{2}}\right)\right| \leqslant \\
& \leqslant\left|X_{r}^{(2, j)}\left(z^{i_{1}}\right)\right|+\left|X_{r}^{(2, j)}\left(z^{i_{2}}\right)\right|+\left|X_{r}^{(2, j)}\left(z^{i_{1}}\right) \cap X_{r}^{(2, j)}\left(z^{i_{2}}\right)\right| \leqslant \\
& \leqslant 3\left|X_{r}^{(2, j)}\left(z^{i_{1}}\right)\right| .
\end{aligned}
$$

Осталось отметить, что

$$
\left|X_{r}^{(2, j)}\left(z^{i_{1}}\right)\right|=C_{n-1}^{r-1}(q-1)^{r}=(r / n) C_{n}^{r}(q-1)^{r} .
$$

Из (62), (63), (69), (70) и условий теоремы 1 вытекает (14).

Теорема 2 доказана.

2016, T. 7, № 4, С. $67-80$ 


\section{Список литературы}

[1] Копытцев В.А., Михайлов В.Г., “Теоремы пуассоновского типа для числа специальных решений случайного линейного включения”, Дискретная математика, 22 :2 (2010), 3-21.

[2] Копытцев В.А., Михайлов В.Г., “Предельные теоремы пуассоновского типа для числа решений обобщенных линейных включений”, Дискретная математика, 24:3 (2012), $103-121$.

[3] Копытцев В.А., “Многомерная теорема Пуассона для чисел решений, близких к заданным векторам, у системы случайных линейных уравнений”, Дискретная математика, 19:4 (2007), 3-22.

[4] Михайлов В.Г., “Сходимость к процессу с независимыми приращениями в схеме нарастающих сумм зависимых случайных величин”, Матем. сб., 94 (1974), 283-299.

[5] Михайлов В.Г., “О предельной теореме Б.А.Севастьянова для сумм зависимых случайных индикаторов”, Обозрение прикл. и промышл. матем., $10: 3$ (2003), 571-578. 